\title{
Understanding New Concepts: Clostridium difficile Infection in Pouch Patients
}

\author{
Grigoriy E. Gurvits
}

Published online: 21 March 2014

(c) Springer Science+Business Media New York 2014

Inflammatory bowel disease (IBD) is a major medical condition that affects nearly 1.5 million Americans, with over 70,000 new cases diagnosed annually. Over the last 50 years, rapid advances in the understanding of its pathophysiology and diagnosis, combined with the utilization of emerging medical therapies have better controlled the disease, have decreased morbidity, and have prolonged survival. Yet, the lifelong risk of surgery in patients with Crohn's disease (CD) was traditionally reported at $75 \%$ and with ulcerative colitis (UC) at $25 \%$, numbers likely to stay unchanged. One of the frequent operations performed for patients with these diseases is restorative proctocolectomy with ileal pouch anal anastomosis (IPAA), a procedure reserved for patients with advanced UC refractory to currently available medical therapy or who develop colonic dysplasia or cancer. First described in 1978 by Parks and Nicholls [1], this procedure truly revolutionized patient care in that it maintained gut continuity and avoided the need for permanent ileostomy in patients with UC. Over the years, with technical improvements, it has also become the procedure of choice for patients with familial adenomatous polyposis syndrome requiring colectomy.

IPAA is not without significant side effects; it is estimated that nearly $40 \%$ of patients will develop acute pouchitis, of which nearly a quarter will recur. Risk factors include concurrent primary sclerosing cholangitis, antecolectomy thrombocytosis, non-smoker status, non-steroidal anti-inflammatory drug use, ischemia, and "backwash" ileitis [2]. The etiology of pouchitis is thought to be a

\section{G. E. Gurvits $(\square)$}

Division of Gastroenterology, New York University School of Medicine/Langone Medical Center, 240 East 38th Street, 23

Floor, New York, NY 10016, USA

e-mail: g_gurvits@hotmail.com combination of genetic, environmental, anatomic, and immunologic factors. Treatment of acute and chronic pouchitis is typically tedious, requiring flexibility with antibiotic and probiotic regimens, frequent diagnostic and therapeutic pouchoscopy, and occasionally the administration of potent immunomodulatory drugs and biologic agents. The spectrum of pouchitis, extending from acute antibiotic responsive to chronic antibiotic refractory, poses a significant challenge to the gastroenterologist: What if any factors can we anticipate and potentially eliminate to delay the progression of the disease? Specifically, will the rising incidence of preoperative Clostridium difficile infection (CDI) in IBD patients have an effect on developing pouchitis postoperatively, and if so, how long will it take to present?

CDI is increasingly included in the differential diagnosis of pouchitis. In fact, CDI occurs in over $10 \%$ of symptomatic patients with IPAA and is an important cause of chronic refractory pouchitis [3]. With global incidence on the rise and increasing attention from the media, this gram negative anaerobe has been increasingly recognized as a factor in IBD patients, whether pre- and postoperative. Increased antibiotic use, alterations of gut microbiota, exposure to health care environment, coupled with immune imbalances place our IBD patients at an increased risk of developing CDI. In this respect, the paper by Dr. Sun et al. in this issue of Digestive Diseases and Sciences [4] provides unique insight into the complex relationship between preoperative CDI and the development of CDI in IPAA patients. At first glance, the authors drew a rather unexpected conclusion: Our "gut feeling" was wrong-preoperative infection is not associated with an increased risk of CDI in ileal pouch patients.

Let's analyze this closely. Clostridium species may asymptomatically colonize the intestine or may lead to 
inflammation with fulminant colitis, accompanied by clinical deterioration. Nearly $20 \%$ of patients with initial CDI will develop symptomatic recurrence, with effectively double the risk for developing future infections [5]. The results of Dr. Sun's study unexpectedly show that this is not necessarily true in the subset of patients who had undergone total proctocolectomy. Pathogenic $C$. difficile is overwhelmingly an infection of the large intestine, with only a handful of case reports of post-surgical small bowel involvement. IBD and its medical management are known risk factors for de-novo and recurrent CDI, and treatment of $C$. difficile in this patient group is challenging, often requiring cautious balancing of established drug combinations [6]. Curative proctocolectomy eliminates UC and with it the need for immunosuppression, frequent antibiotic use, and increased nosocomial exposure to the bacteria. Importantly, this surgery also removes the natural reservoir of the $C$. difficile, a familiar territory where it would assimilate until next recurrence.

As Dr. Sun and his colleagues point out, the median follow-up duration post-IPAA was higher by 2 years in patients who tested positive for CDI compared with the non-infected patients. However, the fact that CDI does eventually develop in IPAA patients raises the question of whether this represents a reinfection with a new strain of the bacteria that colonizes small intestine and progressively overwhelms its defense barriers in the setting of the eventual adaptive colonic metaplasia of the pouch and distal ileum that provides the environment for clinical CDI to develop. Initially, diminished small intestinal receptor activity and absence of toxin-specific binding sites leads to an insignificant biologic response to the CDI toxin production. However, continuous stimulation of the ileal mucosa with $C$. difficile toxin A may increase brush border receptor binding in a time-related fashion [7]. In fact, asymptomatic $C$. difficile colonization occurs in up to $70 \%$ of healthy infants, mostly due to immaturity of the intestine and absence of competing microbiota. This number decreases to $<4 \%$ in the adult population [8]. Finally, types of pouch anatomy (J-pouch, K-pouch, and S-pouch) and post-surgical complications may conceivably predispose to an accelerated colonization of microbial organisms and cellular adaptations required for the presentation of new CDI. Further categorization is necessary, including a separate analysis of small subset of IPAA patients with CD or patients with unrecognized rectal remnant who develop CDI.

So, if patients with UC who underwent restorative proctocolectomy with IPAA eventually become receptive to the development of CDI, what are important factors that get them over the edge? Broad spectrum antibiotics are the mainstay of therapy for initial and recurrent episodes of pouchitis, placing patients at an increased risk for initial CDI. Up to $85 \%$ of the patients with CDI receive antimicrobial therapy within 4 weeks of the onset of symptoms. Continued use of antibiotics remains the largest risk factor for its recurrence [9] and recent data suggests that decreased overall diversity of the microbiota of the gut was associated with increased risk of subsequent bout of CDI [10].

In summary, the study by Sun et al. [1] reports an important lack of correlation between preoperative CDI and an increased risk of postoperative CDI in IPAA patients based on an analysis of date obtained from a tertiary care center pouch clinic. It also invites a number of new questions, thus opening the door to future prospective research studies. With rising prevalence of CDI in the IBD population and clear impact of $C$. difficile on its short- and long-term prognosis [11], we hope to continue advancing our knowledge in search of effective tools in disease prevention and, importantly, its outcome.

\section{Conflict of interest None}

\section{References}

1. Parks AG, Nicholls RJ. Proctocolectomy without ileostomy for ulcerative colitis. BMJ. 1978;2:85-88.

2. Maser EA, Present DH. Pouch-ouch. Curr Opin Gastroenterol. 2008;24:70-74.

3. Li Y, Qian J, Queener E, Shen B. Risk factors and outcome of PCR-detected Clostridium difficile infection in ileal pouch patients. Inflamm Bowel Dis. 2013;19:397-403.

4. Sun C, Du P, Wu XR, Queener E, Shen B. Preoperative Clostridium difficile infection is not associated with an increased risk for the infection in ileal pouch patients. Dig Dis Sci (Epub ahead of print). doi: 10.1007/s10620-014-3047-0.

5. Johnson S. Recurrent Clostridium difficile infection: a review of risk factors, treatments, and outcomes. J Infect. 2009;58:403-410.

6. Ananthakrishnan AN. Detecting and treating Clostridium difficile infections in patients with inflammatory bowel disease. Gastroenterol Clin North Am. 2012;41:339-353.

7. Eglow R, Pothoulakis C, Itzkowitz S, et al. Diminished Clostridium difficile toxin A sensitivity in newborn rabbit ileum is associated with decreased toxin A receptor. J Clin Invest. 1992;90:822-829.

8. Sammons JS, Toltzis P, Zaoutis TE. Clostridium difficile infection in children. JAMA Pediatr. 2013;167:567-573.

9. Garey KW, Sethi S, Yadav Y, DuPont HL. Meta-analysis to assess risk factors for recurrent Clostridium difficile infection. $J$ Hosp Infect. 2008;70:298-304.

10. Chang JY, Antonopoulos DA, Kalra A, et al. Decreased diversity of the fecal microbiome in recurrent Clostridium difficile associated diarrhea. J Infect Dis. 2008;197:435-438.

11. Ananthakrishnan AN, Binion DG. Impact of Clostridium difficile on inflammatory bowel disease. Expert Rev Gastroenterol Hepatol. 2010;4:589-600. 